\title{
Autofluorescent polarimetry in diagnostics of endometriosis and infertility
}

\author{
A.G. Ushenko ${ }^{1}$, G.D. Koval ${ }^{2}$, D.M. Burkovets ${ }^{1}$, V.O. Savich ${ }^{2}$ \\ ${ }^{1}$ Chernivtsi National University, Correlation Optics Department, \\ 2, Kotsyubinsky str., 58012 Chernivtsi, Ukraine \\ ${ }^{2}$ Bukovinian State Medical University, 58000 Chernivtsi, Ukraine \\ E-mail:a.dubolazov@chnu.edu.ua
}

\begin{abstract}
A new information about optical technique in diagnostics for the endometrium structure has been proposed. The model of Mueller-matrix description of mechanisms responsible for optical anisotropy of these objects (optical activity, birefringence as well as linear and circular dichroism) has been suggested. The ensemble of informationally topical azimuthally stable Mueller-matrix invariants has been determined. Using the statistical analysis of the distribution parameters, the objective criteria for differentiation of endometrium taken from healthy women and patients with endometriosis were determined. From the viewpoint of probative medicine, the operational characteristics (sensitivity, specificity and accuracy) of the information-optical method applied to Mueller-matrix mapping of endometrium histological section have been found and its efficiency in diagnostics of endometriosis and infertility has been demonstrated.
\end{abstract}

Keywords: autofluorescence polarimetry, endometrium.

Manuscript received 07.11.14; revised version received 23.02.15; accepted for publication 27.05.15; published online 08.06.15

\section{Introduction}

Nowadays, in biological and medical investigations many practical techniques based on measurements and analysis of Mueller's matrices are used $[1,2]$. In recent 10-15 years, a separate approach - laser polarimetry of histological sections of biological tissues [3] - has been formed in matrix optics. Within this approach, the interactions between the set of statistical (moments of the $1^{\text {st }}$ to $4^{\text {th }}$ orders) [4] and correlation (autocorrelation functions) [5] parameters characterizing Mueller-matrix elements distribution and parameters of linear birefringence of human biological tissues have been determined.

However, laser polarimetry techniques require further development and generalization. First, not all elements of the Mueller matrix prove to be convenient for characterizing biological samples. The reason for it is the azimuthal dependence of the majority of matrix elements - generally 12 of 16 elements change at rotation of the sample around the probing axis. Second, the spectrum of mechanisms of optical anisotropy of biological layers is not confined to linear birefringence only. Taking into consideration the impact of other mechanisms - circular birefringence as well as linear and circular dichroism - appears to be topical in the aspect of enlarging the range of diagnostic techniques. Third, there is a wide range of optically anisotropic biological objects, for which laser polarimetry techniques do not spread widely. The objects of this class are easily accessible and do not require the traumatic surgery of biopsy. Fourth, the methods of laser autofluorescence diagnostics are being progressively developed.

This research is focused on the development of the method of "azimuthally stable" autofluorescence Mueller-matrix mapping of optical anisotropy of endometrium in the task of endometriosis diagnostics. 


\section{Brief theoretical background}

The description of mechanisms responsible for optical anisotropy characteristics of endometrium is based on the following model ideas $[3,9]$ :

1. Crystals of basic proteins of endometrium possess optical activity and are characterized by the matrix operator

$$
\{\Omega\}=\left\|\begin{array}{cccc}
1 & 0 & 0 & 0 \\
0 & \omega_{22} & \omega_{23} & 0 \\
0 & \omega_{32} & \omega_{33} & 0 \\
0 & 0 & 0 & 1
\end{array}\right\|,
$$

where

$$
\omega_{i k}=\left\{\begin{array}{l}
\omega_{22}=\omega_{33}=\cos 2 \theta \\
\omega_{23}=-\omega_{32}=\sin 2 \theta .
\end{array}\right.
$$

Here, $\theta$ is the rotation angle of the polarization plane of the transformed light beam.

2. Networks possess the linear birefringence and linear dichroism. Optical manifestations of such mechanisms are completely described by Mueller matrices:

\subsection{Linear birefringence}

$$
\{D\}=\left\|\begin{array}{cccc}
1 & 0 & 0 & 0 \\
0 & d_{22} & d_{23} & d_{24} \\
0 & d_{32} & d_{33} & d_{34} \\
0 & d_{42} & d_{43} & d_{44}
\end{array}\right\|
$$

where

$$
d_{i k}=\left\{\begin{array}{l}
d_{22}=\cos ^{2} 2 \rho+\sin ^{2} 2 \rho \cos , \\
d_{23}=d_{32}=\cos 2 \rho \sin 2 \rho(1-\cos \delta), \\
d_{33}=\sin ^{2} 2 \rho+\cos ^{2} 2 \rho \cos \delta, \\
d_{24}=-d_{42}=\sin 2 \rho \sin \delta, \\
d_{34}=-d_{43}=\cos 2 \rho \sin \delta, \\
d_{44}=\cos \delta .
\end{array}\right.
$$

Here, $\rho$ is the direction of optical axis of biological crystal, $\delta$ is the phase shift between linearly polarized orthogonal components of light beam amplitude.

\subsection{Linear dichroism}

$$
\{\Psi\}=\left\|\begin{array}{cccc}
1 & \varphi_{12} & \varphi_{13} & 0 \\
\varphi_{21} & \varphi_{22} & \varphi_{23} & 0 \\
\varphi_{31} & \varphi_{32} & \varphi_{33} & 0 \\
0 & 0 & 0 & \varphi_{44}
\end{array}\right\|
$$

where

$$
\varphi_{i k}=\left\{\begin{array}{l}
\varphi_{12}=\varphi_{21}=(1-\Delta \tau) \cos 2 \rho, \\
\varphi_{13}=\varphi_{31}=(1-\Delta \tau) \sin 2 \rho, \\
\varphi_{22}=(1+\Delta \tau) \cos ^{2} 2 \rho+2 \sqrt{\Delta \tau} \sin ^{2} 2 \rho, \\
\varphi_{23}=\varphi_{32}=(1-\Delta \tau) \sin 2 \rho, \\
\varphi_{33}=(1+\Delta \tau) \sin ^{2} 2 \rho+2 \sqrt{\Delta \tau} \cos ^{2} 2 \rho, \\
\varphi_{44}=2 \sqrt{\Delta \tau} .
\end{array}\right.
$$

Here, $\Delta \tau=\frac{\tau_{x}}{\tau_{y}}, \quad\left\{\begin{array}{l}\tau_{x}=\tau \cos \rho ; \\ \tau_{y}=\tau \sin \rho\end{array}, \quad \tau_{x}, \quad \tau_{y} \quad\right.$ are the absorption coefficients of linearly polarized orthogonal components of the light beam amplitude.

3. Availability of complex spiral-like protein molecular structures forms circular dichroism. Optical manifestations of these configuration peculiarities are characterized by the Mueller matrix

$$
\{\Phi\}=\left\|\begin{array}{cccc}
1 & 0 & 0 & \phi_{14} \\
0 & \phi_{22} & 0 & 0 \\
0 & 0 & \phi_{33} & 0 \\
\phi_{41} & 0 & 0 & 1
\end{array}\right\|
$$

where

$$
\phi_{i k}=\left\{\begin{array}{l}
\phi_{22}=\phi_{33}=\frac{1-C^{2}}{1+C^{2}}, \\
\phi_{14}=\phi_{41}= \pm \frac{2 C}{1+C^{2}} .
\end{array}\right.
$$

Here, $C=\frac{g_{\otimes}-g_{\oplus}}{g_{\otimes}+g_{\oplus}}, g_{\otimes}, \quad g_{\oplus}$ are the absorption indices of left- $(\otimes)$ and right-hand $(\oplus)$ circularly polarized components of the light beam amplitude.

Considering the influence of all mechanisms of optical anisotropy, Mueller matrix of endometrium can be written as follows:

$$
\{M\}=\{\Omega\}\{D\}\{\Psi\}\{\Phi\}=\left\|\begin{array}{cccc}
1 & M_{12} & M_{13} & M_{14} \\
M_{21} & M_{22} & M_{23} & M_{24} \\
M_{31} & M_{32} & M_{33} & M_{34} \\
M_{41} & M_{42} & M_{43} & M_{44}
\end{array}\right\| \rightarrow M_{i k} \neq 0 .(9)
$$

It is shown in [10-25] that the following elements of the matrix $\{M\}$ and their combinations are azimuthally stable and independent of the sample rotation angle $\Theta$ :

$$
\begin{aligned}
& \left\{\begin{array}{l}
M_{11}(\Theta)=\text { const, } \\
M_{14}(\Theta)=\text { const, } \\
M_{41}(\Theta)=\text { const, } \\
M_{44}(\Theta)=\text { const, }
\end{array}\right. \\
& \left\{\begin{array}{l}
{\left[M_{22}+M_{33}\right](\Theta) \equiv \Sigma M_{22 ; 33}(\Theta)=\text { const },} \\
{\left[M_{23}-M_{32}\right](\Theta) \equiv \Delta M_{23 ; 32}(\Theta)=\text { const. }}
\end{array}\right.
\end{aligned}
$$

The analysis of the rotation invariants (10), (11) reveals their individual "information" loading. Let us consider this statement in detail. In the visible part of the spectrum, the manifestations of phase anisotropy predominate in the forms of linear and circular birefringence. Extreme values of the parameters of linear $(\Delta \tau)$ and circular dichroism $(C)$ of proteins lie in the ultraviolet spectral range. In other words,

$$
\delta, \theta \gg \Delta \tau, C \text {. }
$$

Considering (12), the matrix operator (9) can be rewritten as 


$$
\{M(\rho, \delta, \theta)\}=\left\|\begin{array}{|cccc}
1 & 0 & 0 & 0 \\
0 & \left(d_{22} \omega_{22}+d_{23} \omega_{32}\right) & \left(d_{22} \omega_{23}+d_{23} \omega_{33}\right) & d_{24} \\
0 & \left(d_{32} \omega_{22}+d_{33} \omega_{32}\right) & \left(d_{32} \omega_{23}+d_{33} \omega_{33}\right) & d_{34} \\
0 & \left(d_{42} \omega_{22}+d_{43} \omega_{32}\right) & \left(d_{42} \omega_{23}+d_{43} \omega_{33}\right) & d_{44}
\end{array}\right\| \equiv\left\|\begin{array}{cccc}
1 & 0 & 0 & 0 \\
0 & M_{22} & M_{23} & M_{24} \\
0 & M_{32} & M_{33} & M_{34} \\
0 & M_{42} & M_{43} & M_{44}
\end{array}\right\| .
$$

It follows from (13) that the values of Muellermatrix rotation invariants $M_{44}(\Theta), \frac{\Delta M_{23 ; 32}(\Theta)}{\sum M_{22 ; 33}(\Theta)}$ are generally determined by the parameters of linear $(\delta)$ and circular $(\theta)$ birefringence

$$
\left\{\begin{array}{l}
M_{44} \sim \cos \delta \\
\frac{\Delta M_{23 ; 32}}{\sum M_{22 ; 33}} \sim \tan \theta .
\end{array}\right.
$$

Despite the lower level of manifestations of the effects of anisotropic absorption of laser radiation (Eqs. (5)-(8)), some Mueller matrix elements appear to be sensitive to these mechanisms. At the presence of linear dichroism $\Delta \tau$, the matrix operator (9) is transformed into the expression

$$
\begin{aligned}
& \{M(\rho, \delta, \theta, \Delta \tau)\}= \\
& =\left\|\begin{array}{cccc}
1 & \left(\varphi_{12} t_{22}+\varphi_{13} t_{32}\right) & \left(\varphi_{12} t_{23}+\varphi_{13} t_{33}\right) & \left(\varphi_{12} t_{24}+\varphi_{13} t_{34}\right) \\
\varphi_{21} & \left(\varphi_{22} t_{22}+\varphi_{23} t_{32}\right) & \left(\varphi_{22} t_{23}+\varphi_{32} t_{33}\right) & \left(\varphi_{22} t_{24}+\varphi_{32} t_{34}\right) \\
\varphi_{31} & \left(\varphi_{32} t_{22}+\varphi_{33} t_{32}\right) & \left(\varphi_{32} t_{23}+\varphi_{33} t_{33}\right) & \left(\varphi_{32} t_{24}+\varphi_{33} t_{34}\right) \\
0 & \varphi_{44} t_{42} & \varphi_{44} t_{43} & \varphi_{44} t_{44}
\end{array}\right\| .
\end{aligned}
$$

Comparison of (13) and (15) shows that the condition $M_{14}(\Theta) \neq 0$ is the criterion of the presence of linear dichroism:

$M_{14}=\varphi_{12} d_{24}+\varphi_{13} d_{34}=(1-\Delta \tau) \sin \delta$.

Circular dichroism $C$ is manifested unlike zero of rotation invariant $M_{41}\left(\lambda_{2}\right) \neq 0$

$M_{41}=\varphi_{44} d_{44} c_{41}=4 \sqrt{\Delta \tau} \cos \delta \frac{C}{1+C^{2}}$.

Thus, having measured the parameter distributions $q \equiv\left\{\begin{array}{l}M_{14 ; 41 ; 44}(m \times n) \\ \Delta M_{i k}=\frac{\Delta M_{23 ; 32}}{\sum M_{22 ; 33}}(m \times n), \quad \text { one can obtain }\end{array}\right.$ azimuthally stable information about optical anisotropy of endometrium.

\section{Investigation technique and processing of Mueller-matrix images}

The measurements of coordinate distributions of Mueller-matrix elements were performed in the setup of the standard Stokes-polarimeter [3]. For excitation, we used autofluorescence emission of blue $(\lambda=0.405 \mu \mathrm{m})$ solid-state laser.

The Stokes-vector parameters $S_{i=2 ; 3 ; 4}^{0 ; 4 ; 90 ; \otimes}$ were measured in the points $(m \times n)$ of the digital image

$\left\{\begin{array}{l}S_{i=2}^{0 ; 45 ; 90 ; \otimes}=I_{0}{ }^{0 ; 45 ; 90 ; \otimes}-I_{90}{ }^{0 ; 45 ; 90 ; \otimes} \\ S_{i=3}^{0 ; 45 ; 90 ; \otimes}=I_{45}^{0 ; 45 ; 90 ; \otimes}-I_{135}^{0 ; 45 ; 90 ; \otimes} \\ S_{i=4}^{0 ; 45 ; 90 ; \otimes}=I_{\otimes}{ }^{0 ; 45 ; 90 ; \otimes}-I_{\oplus}{ }^{0 ; 45 ; 90 ; \otimes}\end{array}\right.$.

Here $I_{0 ; 90 ; 55 ; 135 ; \otimes ; \oplus}^{0 ; 45 ; 0}$ are the intensities of linearly $\left(0^{\circ}, 90^{\circ}, 45^{\circ}, 135^{\circ}\right)$, right- $(\otimes)$ and left-hand $(\oplus)$ circularly polarized components of filtered (using the polarizer 9 and quarter-wave plate 8 ) laser radiation.

Further, the "informationally topical" parameters (10), (11) were calculated via the algorithm:

$$
\left\{\begin{array}{l}
M_{44}=S_{4}^{\otimes}-0.5\left(S_{4}^{0}+S_{4}^{90}\right), \\
\sum_{22 ; 33}=M_{22}+M_{33}=0.5\left(S_{2}^{0}-S_{2}^{90}\right)+S_{3}^{45}-0.5\left(S_{3}^{0}+S_{3}^{90}\right), \\
\Delta M_{23 ; 32}=M_{23}-M_{32}=S_{2}^{45}-0.5\left(S_{2}^{0}+S_{2}^{90}\right)-0.5\left(S_{3}^{0}-S_{3}^{90}\right) .
\end{array}\right.
$$

For the objective assessment of coordinate distributions $q \equiv\left\{\begin{array}{l}M_{14 ; 41 ; 44}(m \times n), \\ \frac{\Delta M_{23 ; 32}}{\sum M_{22 ; 33}}(m \times n)\end{array}\right.$ we applied the synthesis of traditional methods of statistical and correlation analyses.

A set of statistical moments of the $1^{\text {st }}$ to $4^{\text {th }}$ orders characterizing distributions $q$ was calculated using the following algorithms:

$Z_{1}=\frac{1}{N} \sum_{j=1}^{N}|q|_{j} ; Z_{2}=\sqrt{\frac{1}{N} \sum_{j=1}^{N}(q)_{j}^{2}} ;$

$Z_{3}=\frac{1}{Z_{2}^{3}} \frac{1}{N} \sum_{j=1}^{N}(q)_{j}^{3} ; Z_{4}=\frac{1}{Z_{2}^{4}} \frac{1}{N} \sum_{j=1}^{N}(q)_{j}^{4}$,

where $N$ is the number of pixels of CCD-camera.

\section{Analysis and discussion of experimental data}

Optically thin (extinction index $\tau<0.1$ ) donor endometrium dried at room temperature (group $1-25$ patients) and those of pathology (group $2-25$ patients) were used as objects of investigation. Figs. 1 to 4 present the series of experimentally measured Mueller-matrix images $M_{i k}(m \times n)$ (relations (19)). 


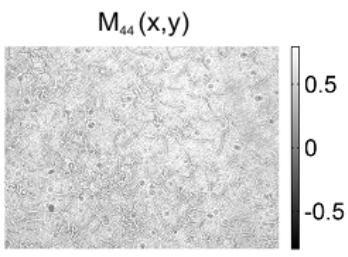

(a)

$\mathrm{M}_{44}(\mathrm{x}, \mathrm{y})$

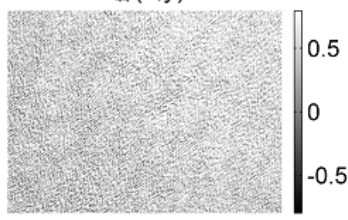

(c)

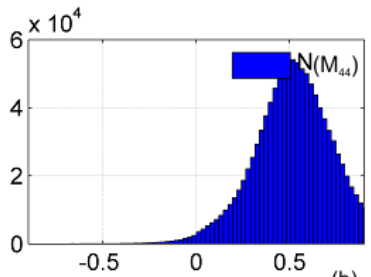

(b)

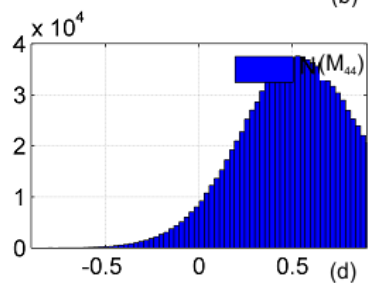

Fig. 1. Coordinate distributions of the Mueller-matrix rotation invariant $M_{44}$ characterizing linear birefringence of donor endometrium $(a-b)$ and of patients with endometriosis $(c-d)$.

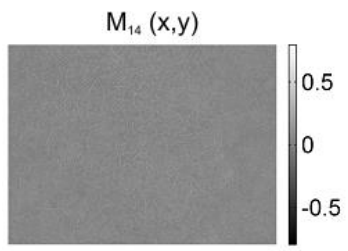

(a)

$\mathrm{M}_{14}(\mathrm{x}, \mathrm{y})$

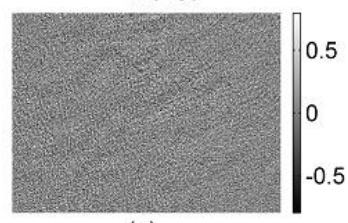

(c)
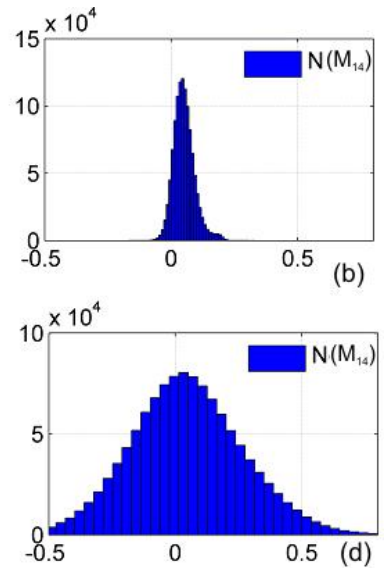

Fig. 2. Coordinate distributions of the Mueller-matrix rotation invariant $M_{14}$ characterizing linear dichroism of donor endometrium $(a-b)$ and of patients with endometriosis $(c-d)$.

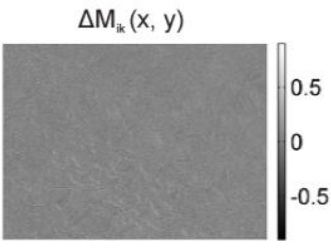

(a)

$\Delta \mathrm{M}_{\mathrm{ik}}(\mathrm{x}, \mathrm{y})$

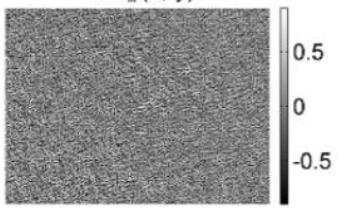

(c)
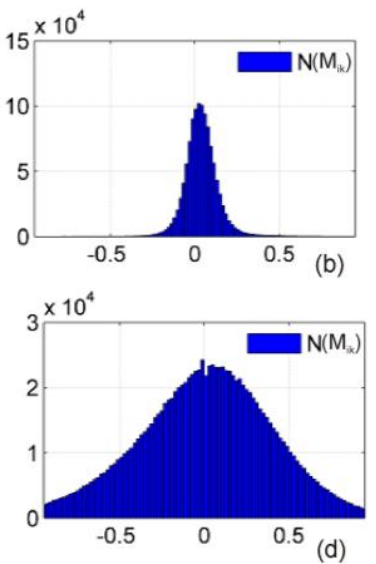

Fig. 3. Coordinate distributions of the Mueller-matrix rotation invariant $\Delta M_{i k}$ characterizing circular birefringence of donor endometrium $(a-b)$ and of patients with endometriosis $(c-d)$.

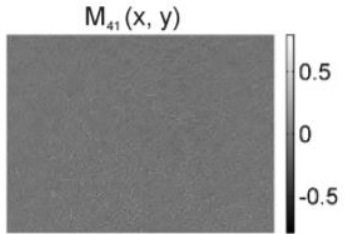

(a)

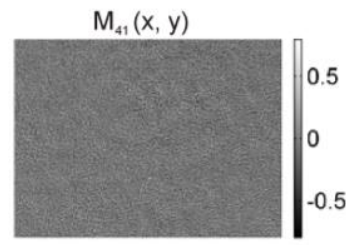

(c)
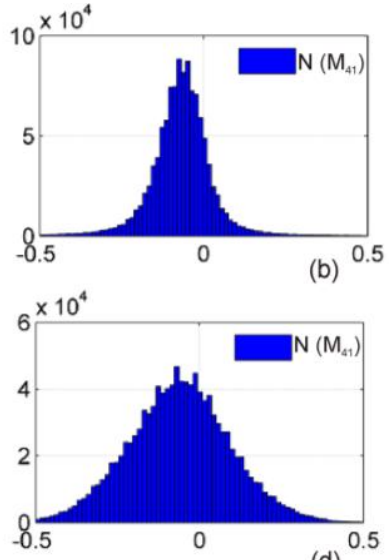

(d)
Fig. 4. Coordinate distributions of the Mueller-matrix rotation invariant $M_{41}$ characterizing circular dichroism of donor endometrium $(a-b)$ and of patients with endometriosis $(c-d)$.

The comparative analysis of the obtained data shows:

- Linear birefringence (Fig. 1). Distributions $M_{44}(m \times n)$ found for the samples of both types are characterized by commensurable range of the values change and rather identical coordinate structure. This fact can be related to that close in values to phase shifts $\delta$ determined by simultaneous contribution of the birefringence value and transverse sizes of endometrium.

- Linear dichroism (Fig. 2). It is determined that the network in donor endometrium has a greater level of linear dichroism in comparison with similar sample of patient with endometriosis. The differences found between Mueller-matrix images $M_{14}(m \times n)$ can be related to the differences in proteins of endometrium. For the sample from group 1, a greater concentration of albumin is typical. It is observed in formation of the network of spatially oriented lathlike crystals. On the contrary, the increase of concentration of optically active globulin leads to formation of the restructured network with minimal linear dichroism.

- Circular birefringence (Fig. 3). It is defined that distributions $\Delta M_{i k}$ of the endometrium of patients with endometriosis exceed the similar dependences for the samples from the group 1.

- $\quad$ Circular dichroism (Fig. 4). Optically anisotropic manifestations of the tertiary structure of endometrium are more vividly pronounced in a healthy person. The range of changes of the value of matrix element $M_{41}$ in the sample from the group 1 are by 2-3 times higher than in Muellermatrix image of the sample from the group 2 .

The results of quantitative statistical (relations (19)) and correlation (relations (20), (21)) analysis of a series of Mueller-matrix rotational invariants of two groups of endometrium are illustrated by the data presented in Table 1. 
Table 1. Statistical $\left(Z_{i=1 ; 2 ; 3 ; 4}\right)$ moments of distributions of Mueller-matrix invariants of endometrium of group 1 (normal) and group 2 (endometriosis).

\begin{tabular}{|c|c|c|c|c|c|c|c|c|}
\hline \multirow{2}{*}{$\begin{array}{c}\text { Para- } \\
\text { meters }\end{array}$} & \multicolumn{2}{|c|}{$M_{44}$} & \multicolumn{2}{|c|}{$M_{14}$} & \multicolumn{2}{c|}{$\Delta M_{i k}$} & \multicolumn{2}{c|}{$M_{41}$} \\
\cline { 2 - 9 } & Normal & $\begin{array}{c}\text { Endo- } \\
\text { metriosis }\end{array}$ & Normal & $\begin{array}{c}\text { Endo- } \\
\text { metriosis }\end{array}$ & Normal & Endometriosis & Normal & Endometriosis \\
\hline$Z_{1}$ & $0.34 \pm 0.061$ & $0.26 \pm 0.057$ & $0.18 \pm 0.038$ & $0.11 \pm 0.011$ & $0.12 \pm 0.024$ & $0.18 \pm 0.038$ & $0.18 \pm 0.018$ & $0.09 \pm 0.011$ \\
\hline$Z_{2}$ & $0.16 \pm 0.018$ & $0.19 \pm 0.024$ & $0.22 \pm 0.033$ & $0.15 \pm 0.019$ & $0.15 \pm 0.019$ & $0.11 \pm 0.021$ & $0.13 \pm 0.046$ & $0.11 \pm 0.018$ \\
\hline$Z_{3}$ & $0.23 \pm 0.043$ & $0.31 \pm 0.061$ & $0.78 \pm 0.14$ & $1.31 \pm 0.39$ & $0.79 \pm 0.12$ & $0.53 \pm 0.059$ & $1.27 \pm 0.15$ & $2.81 \pm 0.53$ \\
\hline$Z_{4}$ & $0.29 \pm 0.029$ & $0.22 \pm 0.038$ & $0.88 \pm 0.11$ & $2.14 \pm 0.47$ & $0.68 \pm 0.16$ & $0.44 \pm 0.065$ & $1.02 \pm 0.22$ & $2.59 \pm 0.73$ \\
\hline
\end{tabular}

Table 2. Operational characteristics of Mueller-matrix mapping techniques.

\begin{tabular}{|c|c|c|c|c|}
\hline Parameters & $M_{44}$ & $M_{14}$ & $\Delta M_{i k}$ & $M_{41}$ \\
\hline$S e\left(Z_{i}\right), \%$ & 75 & 86 & 77 & 88 \\
\hline$S p\left(Z_{i}\right), \%$ & 66 & 77 & 74 & 71 \\
\hline$A c\left(Z_{i}\right), \%$ & 71 & 79 & 82 & 86 \\
\hline
\end{tabular}

Table 3. Operational characteristics of Mueller-matrix mapping techniques.

\begin{tabular}{|c|c|c|c|c|}
\hline Parameters & liver & spleen & kidney & pancreas \\
\hline $\operatorname{Se}\left(Z_{i}\right), \%$ & 74 & 81 & 87 & 68 \\
\hline $\operatorname{Sp}\left(Z_{i}\right), \%$ & 69 & 74 & 78 & 73 \\
\hline$A c\left(Z_{i}\right), \%$ & 72 & 77 & 84 & 71 \\
\hline
\end{tabular}

The most sensitive parameters for differentiation of optically anisotropic networks of endometrium in both groups are highlighted with grey color in Table 1.

For possible clinical application of Mueller-matrix mapping within the investigated groups of samples, the operational characteristics typical for probative medicine are: $\quad$ sensitivity $\quad S e=\frac{a}{a+b} 100 \%, \quad$ specificity $S p=\frac{c}{c+d} 100 \%$ and accuracy $A c=\frac{S e+S p}{2}$, where $a$ and $b$ are the numbers of correct and wrong diagnoses within the group $1 ; c$ and $d$ is the same within the group 2 (Table 2).

Thus, the statistical and correlation analyses of Mueller-matrix invariants characterizing polarization manifestations of linear dichroism, circular birefringence and circular dichroism appeared to be efficient in the task of differential diagnostics of endometriosis.

This method was tested dealing with the problem of differentiation of histological sections of the internal organs of healthy and diabetic rats (liver, spleen, kidney and pancreas) - Table 3 .

\section{Conclusions}

1. The Mueller-matrix invariants characterizing polarization manifestations of partial mechanisms proving optical anisotropy - linear birefringence, linear dichroism, circular birefringence and circular dichroism of endometrium - have been determined.

2. Within the statistical approach, the interrelations between the set of moments of the $1^{\text {st }}$ to $4^{\text {th }}$ orders and peculiarities of optically anisotropic networks of endometrium taken from healthy women and women with endometriosis have been determined.

\section{Acknowledgement}

This work was supported by the grants № 0113 U003239 and № 0112 U002336 from the Ukrainian Foundation for Basic Researches.

\section{References}

1. A.G. Ushenko, V.P. Pishak, Laser polarimetry of biological tissue: Principles and applications, in: Handbook of Coherent-Domain Optical Methods: Biomedical Diagnostics, Environmental and Material Science, 1, p. 93-138 (2004).

2. O.V. Angelsky, A.G. Ushenko, Yu.A. Ushenko, V.P. Pishak, A.P. Peresunko, Statistical, correlation and topological approaches in diagnostics of the structure and physiological state of birefringent biological tissues, in: Handbook of Photonics for Biomedical Science, p. 283-322 (2010).

3. Y.A. Ushenko, T.M. Boychuk, V.T. Bachynsky, O.P. Mincer, Diagnostics of structure and physiological state of birefringent biological tissues: Statistical, correlation and topological approaches, in: Handbook of Coherent-Domain Optical Methods, p. 107-148 (2013).

4. Yu.A. Ushenko, G.B. Bodnar, G.D. Koval, Classifying optical properties of surface-and bulkscattering biological layers with polarization singular states // J. Innov. Opt. Health Sci. 6, 1350018 (2013).

5. Yu.A. Ushenko, Statistical structure of polarization-inhomogeneous images of biotissues with different morphological structures // Ukr. J. Phys. Opt. 6, p. 63-70 (2005).

6. Yu.A. Ushenko, A.P. Peresunko, B.A. Baku, A new method of Mueller-matrix diagnostics and 
differentiation of early oncological changes of the skin derma // Adv. Opt. Technol. 2010, 952423 (2010).

7. Yu.A. Ushenko, O.I. Telenga, A.P. Peresunko, O.K. Numan, New parameter for describing and analyzing the optical-anisotropic properties of biological tissues // J. Innov. Opt. Health Sci. 4, p. 463-475 (2011).

8. Yu.A. Ushenko, The feasibilities of using the statistical, fractal and singular processing of hominal blood plasma phase images during the diagnostics and differentiation of mammary gland pathological states // J. Innov. Opt. Health Sci. 5, 1150001 (2012).

9. S.N. Savenkov, V.V. Marienko, E.A. Oberemok, O.I. Sydoruk, Generalized matrix equivalence theorem for polarization theory // Phys. Rev. E, 74, p. 605-607 (2006).

10. M. Shribak, R. Oldenbourg, Techniques for fast and sensitive measurements of two-dimensional birefringence distributions // Appl. Opt. 42, p. 3009-3017 (2003).

11. M.H. Smith, Interpreting Mueller matrix images of tissues // Proc. SPIE, 4257, p. $82-89$ (2001).

12. S. Lu, R.A. Chipman, Interpretation of Mueller matrices based on polar decomposition // J. Opt. Soc. Am. A, 13, p. 1106-1113 (1996).

13. O.V. Angelsky, P.V. Polyanskii, C.V. Felde, The emerging field of correlation optics // Optics and Photonics News, 23(4), p. 25-29 (2012).

14. O.V. Angelsky, A.G. Ushenko, D.N. Burcovets, Yu.A. Ushenko, Polarization visualization and selection of biotissue image two-layer scattering medium // J. Biomed. Opt. 10(1), 014010 (2005).

15. A.Ya. Bekshaev, O.V. Angelsky, S.G. Hanson, C.Yu. Zenkova, Scattering of inhomogeneous circularly polarized optical field and mechanical manifestation of the internal energy flows // Phys. Rev. A, 86, 023847 (2012).

16. O.V. Angelsky, A.Ya. Bekshaev, P.P. Maksimyak, A.P. Maksimyak, S.G. Hanson, C.Yu. Zenkova, Self-diffraction of continuous laser radiation in a disperse medium with absorbing particles // Opt. Exp. 21(7), p. $8922-8938$ (2013).

17. O.V. Angelsky, S.G. Hanson, A.P. Maksimyak, P.P. Maksimyak, On the feasibility for determining the amplitude zeroes in polychromatic fields // Opt. Exp. 13(12), p. 4396-4405 (2005).
18. O.V. Angelsky, R.N. Besaha, I.I. Mokhun, Appearance of wavefront dislocations under interference among beams with simple wavefronts // Optica Applicata, 27(4), p. 272-278 (1997).

19. O.V. Angelsky, A.Ya. Bekshaev, P.P. Maksimyak, A.P. Maksimyak, S.G. Hanson, C.Yu. Zenkova, Orbital rotation without orbital angular momentum: mechanical action of the spin part of the internal energy flow in light beams // Opt. Exp. 20(4), p. 3563-3571 (2012).

20. O.V. Angelsky, G.V. Demianovsky, A.G. Ushenko, D.N. Burkovets, Y.A. Ushenko, Wavelet analysis of two-dimensional birefringence images of architectonics in biotissues for diagnosing pathological changes // J. Biomed. Opt. 9(4), p. 679-690 (2004).

21. O.V. Angel'skiř, A.G. Ushenko, S.B. Ermolenko, D.N. Burkovets, Yu.A. Ushenko, O.V. Pishak, Polarization-based visualization of multifractal structures for the diagnostics of pathological changes in biological tissues // Optika $i$ Spektroskopiya, 89(5), p. 799-804 (2000), in Russian.

22. Yu.O. Ushenko, Yu.Ya. Tomka, O.V. Dubolazov, V.O. Balanets'ka, A.V. Karachevtsev, A.P. Angelsky, Wavelet-analysis for laser images of blood plasma // $A E C E$ - Adv. in Electr. and Comput. Eng., 11(2), (2011).

23. V.T. Bachinsky, Yu.O. Ushenko, Yu.Ya. Tomka, O.V. Dubolazov, V.O. Balanets'ka, A.V. Karachevtsev, Wavelet analysis for polarization maps of networks formed by liquid biological crystals in blood plasma: statistical and fractal approaches // Semiconductor Physics, Quantum Electronics \& Optoelectronics, 13(2), p. 189-201 (2010).

24. Yu.A. Ushenko, Yu.Ya. Tomka, A.V. Dubolazov, V.A. Balanetskaya, V.P. Unguryan, N.I. Zabolotna, B.P. Oleinichenko, Mueller-matrix diagnostics of optical properties inherent to polycrystalline networks of human blood plasma // Semiconductor Physics, Quantum Electronics \& Optoelectronics, 14(1), p. 98-105 (2011).

25. Yu.O. Ushenko, O.V. Dubolazov, A.O. Karachevtsev, M.P. Gorsky, Yu.F. Marchuk, Wavelet analysis of Fourier polarized images of the human bile // Appl. Opt. 51, p. C133-C139 (2012). 